\title{
Under Pressure: Comparison of Aspiration Techniques for Endovascular Mechanical Thrombectomy
}

\author{
(D) Nikoubashman, (DD. Wischer, (D) H.M. Hennemann, (D). Büsen, (D) C. Brockmann, and (D) M. Wiesmann
}

\begin{abstract}
BACKGROUND AND PURPOSE: Blood flow should be interrupted during mechanical thrombectomy to prevent embolization of clot fragments. The purpose of our study was to provide a handy overview of the most common aspiration devices and to quantify their flow characteristics.
\end{abstract}

MATERIALS AND METHODS: We assessed volumetric flow rates generated by a 60-mL VacLok vacuum pressure syringe, a Pump MAX aspiration pump, and a Dominant Flex suction pump connected to the following: 1) an 8F long sheath, 2) an 8F balloon-guide catheter, 3) an ACE 64 distal aspiration catheter, and 4) an AXS Catalyst 6 Distal Access Catheter. We used a water/glycerol solution, which was kept at a constant temperature of $20^{\circ} \mathrm{C}$ (viscosity, $3.7 \mathrm{mPa} \cdot \mathrm{s}$ ).

RESULTS: Aspiration with the syringe and the Dominant Flex suction pump achieved the highest flows, whereas aspiration with the Pump MAX was significantly lower $(P<.001)$. Resistors in the aspiration system (tubing, connectors, and so forth) restricted flows, especially when the resistance of the catheter was small (due to its large diameter) and the connected resistors became the predominant resistance $(P<$ $.001)$. The syringe achieved an average vacuum pressure of $-90 \mathrm{kPa}$, and the resulting flow was constant during almost the entire procedure of filling the syringe.

CONCLUSIONS: Sixty-milliliter VacLok vacuum pressure syringes and the Dominant Flex suction pump achieved high and constant flows likely sufficient to reverse blood flow during thrombectomy with an 8F sheath or balloon-guide catheter in the ICA and modern distal aspiration catheters in the MCA. The Pump MAX aspiration pump is dedicated for use with distal aspiration catheters and is unlikely to reverse blood flow in the ICA and MCA without balloon protection.

$E$ ndovascular mechanical thrombectomy is the most effective treatment option for acute ischemic stroke caused by largevessel occlusion. ${ }^{1}$ In past years, the focus of neurointerventional stroke research has shifted from establishing the method to finding the optimal treatment technique. ${ }^{2-6}$ Effective blood flow management during the procedure is key to safety and effectiveness. ${ }^{7}$ Chueh et $\mathrm{al}^{2}$ have shown that there are thousands of small clot fragments during thrombectomy that may occlude small arterioles and capillaries. Even though small infarctions may not be

Received September 20, 2017; accepted after revision January 31, 2018

From the Department of Diagnostic and Interventional Neuroradiology (O.N., D.W., H.M.H., M.W.), RWTH Aachen University Hospital, Aachen, Germany; Institute of Applied Medical Engineering (M.B.), RWTH Aachen University, Aachen, Germany; and Department of Neuroradiology (C.B.), University Medical Centre, Johannes Gutenberg University, Mainz, Germany.

Please address correspondence to Omid Nikoubashman, MD, Klinik für Neuroradiologie, Universitätsklinikum Aachen, Pauwelsstr 30, 52074 Aachen, Germany,

e-mail: onikoubashman@ukaachen.de

三 Indicates article with supplemental on-line table.

http://dx.doi.org/10.3174/ajnr.A5605 visible on $\mathrm{MR}$ imaging, they can have an important clinical impact. ${ }^{8,9}$ Hence, antegrade blood flow should be interrupted during mechanical thrombectomy to prevent embolization of clot fragments. ${ }^{7}$ For this goal, blood is usually aspirated with syringes or a dedicated aspiration pump proximally through the access catheter and/or distally through an intermediate catheter.

Techniques for flow reversal differ considerably, ranging from pump aspiration through small (5F) guide catheters in the internal carotid artery to syringe aspiration through balloon-guide catheters. Syringes have the advantage of cost-effectiveness and, according to some interventionalists, of subjective feedback of aspiration force when they draw the syringe. However, the actual vacuum pressure is not indicated, and comparably small volumes (usually $\leq 60 \mathrm{~mL}$ ) restrict aspiration duration and may necessitate the use of multiple syringes, which can be impractical during an acute stroke intervention. Aspiration pumps on the other hand allow pressure-controlled and comparably comfortable aspiration. However, their comparably high cost restricts their availability in many hospitals. The choice between a syringe and aspiration 
pump is usually up to the interventionalist's discretion. However, there is not only no consensus about which method is best but also only a little knowledge about the specific characteristics of each method. For instance, the applied vacuum pressure of aspiration pumps is indicated, but the vacuum pressure of a syringe is not. Furthermore, although the flow through a bare catheter can be calculated using the Hagen-Poiseuille equation, in practice, it is considerably more challenging to determine the effective flow after accounting for resistors such as additional valves and connecting tubes between catheters and pumps. This issue is especially problematic because pump distributors do not indicate the resistance of their tubing.

Because mechanical thrombectomy is about to become more common, many interventionalists are going to deal with the conundrum of which technique to choose. Therefore, the purpose of our study was to provide a handy overview of the most common aspiration devices and to quantify flow characteristics and aspiration volumes through proximal and distal access catheters with a syringe and the 2 aspiration pumps that are currently available on the market (Pump MAX aspiration pump; Penumbra, Alameda, California; and Dominant Flex suction pump; Medela, Baar, Switzerland).

\section{MATERIALS AND METHODS \\ Experiment}

We assessed the volumetric flow rate (simply referred to as "flow" in the article) generated by a $60-\mathrm{mL}$ VacLok vacuum pressure syringe (Merit Medical Systems, South Jordan, Utah), a Penumbra Pump MAX aspiration pump, and a Medela Dominant Flex suction pump, connected to the following: 1) an $8 \mathrm{~F}$ long sheath (Shuttle Select; Cook, Bloomington, Illinois), 2) an 8F balloonguide catheter (FlowGate ${ }^{2}$; Stryker Neurovascular, Kalamazoo, Michigan), 3) an 0.064-inch ACE 64 distal aspiration catheter (Penumbra), and 4) a 0.06-inch AXS Catalyst 6 Distal Access Catheter (Stryker Neurovascular). We connected catheters and aspiration devices with a 3-way valve (Discofix; Braun, Melsungen, Germany) and a hemostatic Y-adapter (Gateway Plus; Boston Scientific, Fremont, California) using the tubing of the manufacturer. Syringes were connected directly to the 3 -way valve. No additional resistors (tubing, air filters, and so forth) were added to achieve the highest possible flow.

To simulate the rheologic properties of blood at body temperature, we aspirated a solution of glycerol and water, which was kept at a constant temperature of $20^{\circ} \mathrm{C}$ to ensure a viscosity of 3.7 $\mathrm{mPa} \cdot \mathrm{s}$ and a density of $1.11 \mathrm{~g} / \mathrm{mL} .{ }^{10}$ We measured aspiration flow with pumps as reported previously: We aspirated the solution, which was kept in a reservoir, and assessed the weight of the aspirated fluid in grams. The results of 7 measurements were averaged and converted to a volumetric dimension (milliliters). The aspiration flow (milliliters/second) was obtained by dividing the collected volume by the aspiration time. ${ }^{5}$ Aspiration with a syringe was assessed as aspirated volume with time. For measurements through the sheath and balloon catheter, we used an ultrasonic flow meter (HT110 Bypass Flowmeter \& Tubing Sensor; Transonic, Ithaca, New York), due to the high flow through these catheters. The Penumbra pump was set on the recommended vacuum pressure of -25.5 inches $\mathrm{Hg}(\approx-86.4 \mathrm{kPa})$. The Medela

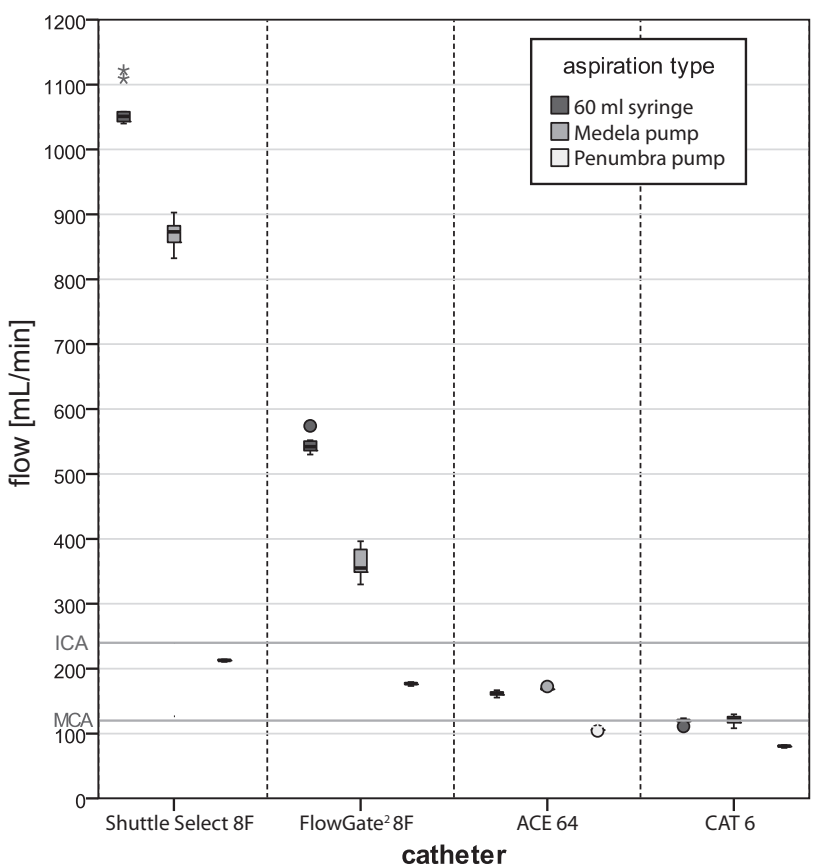

FIG 1. Flow through an $8 \mathrm{~F}$ long sheath (Shuttle Select), an $8 \mathrm{~F}$ ballooncatheter (FlowGate ${ }^{2}$ Balloon Guide Catheter), an ACE 64 distal aspiration catheter, and an AXS Catalyst 6 Distal Access Catheter. Left, Syringe; middle, Medela pump; right, Penumbra pump. Dark gray horizontal lines mark the blood flow in the internal carotid artery and the middle cerebral artery in an average patient.

pump was set on a comparable pressure of $-86.0 \mathrm{kPa}$. Measurements were repeated 7 times. The pressure generated with the syringe and the pressure loss from maximal pressure with time was recorded in 20 measurements using an analog manometer (class 1.6; Riegler Customer Solutions, Bad Urach, Germany).

\section{Statistical Analysis}

We compared experimental results with each other using the ANOVA test, and we compared experimental results with ideal values using a Student $t$ test. $P$ values of an $\alpha$ level $\leq .05$ were defined as significant. All statistical analyses were performed with SPSS 23 software (IBM, Armonk, New York).

\section{RESULTS}

The results of our flow measurements are summarized in the Online Table and Fig 1. Aspiration with the syringe and the Medela pump achieved the highest flows, whereas aspiration flow with the Penumbra pump was significantly lower. Subtotal vacuum and, first and foremost, the resistors in the aspiration system (tubing, connectors, and so forth) restricted flows, in particular when the resistance of the catheter was small (due to its large diameter) and the connected resistors became the predominant resistance (Online Table and Fig 1). The experimentally determined resistance of the Y-adapter and 3-way valve was approximately $212 \times 10^{6}$ $\mathrm{Pa} \cdot \mathrm{s} / \mathrm{m}^{3}$ and $1406 \times 10^{6} \mathrm{~Pa} \cdot \mathrm{s} / \mathrm{m}^{3}$, respectively.

\section{Syringe}

A fully drawn $60-\mathrm{mL}$ syringe achieved a relatively high maximum average vacuum pressure of $-90 \mathrm{kPa}$. Drawing approximately 7 and $20 \mathrm{~mL}$ achieved approximately $-50 \mathrm{kPa}$ and $-75 \mathrm{kPa}$, respectively. Flow was constant during almost the entire procedure 


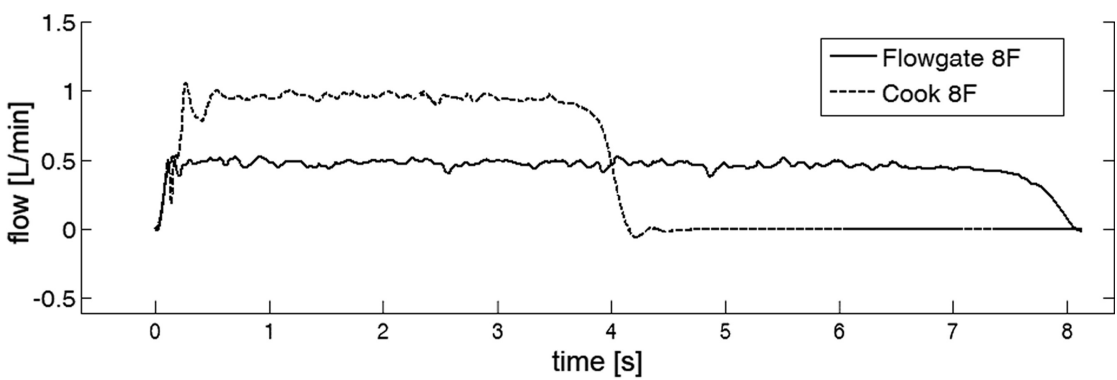

FIG 2. Exemplary flow measurements through an $8 \mathrm{~F}$ long Shuttle Select sheath and an $8 \mathrm{~F}$ FlowGate ${ }^{2}$ balloon catheter with a syringe. Note that the flow is comparably constant and the flow decrease occurs within approximately half a second before the syringe has completely filled. Syringes achieve a comparably constant flow despite the unavoidable pressure loss during filling because it is not the pressure loss that predominantly restricts flow but the resistors (syringe tip, 3 -way valve, and hemostatic valve).

of filling the syringe (Fig 2). Consequently, the syringe achieved comparably high flows. As anticipated, aspiration duration was limited due to high flow on the one hand and restricted syringe volume on the other. The resistance of the narrow tip of the syringe, as well as the connected $\mathrm{Y}$-adapter and 3-way valve, reduced flows significantly $(P<.001)$. When the syringe was drawn but no aspiration was performed, the pressure decreased (from $-90 \mathrm{kPa}$ to $-60 \mathrm{kPa}$ ) by $10 \mathrm{kPa}$ every 91 seconds or $6.6 \mathrm{kPa}$ per minute on average, due to air leakage through the valves and connections.

\section{Aspiration Pumps}

The Medela pump achieved high flow, mostly due to the low resistance of the connecting tubing $\left(3251 \times 10^{6} \mathrm{~Pa} \cdot \mathrm{s} / \mathrm{m}^{3}\right)$. Despite a similarly high vacuum pressure, the Penumbra pump achieved significantly lower flow in all experiments $(P<.001)$, mostly due to the high resistance of the connecting tubing, which was $18,776 \times 10^{6} \mathrm{~Pa} \cdot \mathrm{s} / \mathrm{m}^{3}$ and thus almost 6 times higher than the resistance of the Medela tubing. Notably, the resistance of the old model of the Penumbra tubing $\left(23,756 \times 10^{6} \mathrm{~Pa} \cdot \mathrm{s} / \mathrm{m}^{3}\right)$, which was distributed until 2016 , was approximately $20 \%$ higher compared with the new tubing and resulted in significantly lower flows $(P<.001)$.

\section{DISCUSSION}

Syringes and pumps have been established as basic tools used during mechanical thrombectomy. Aspiration with syringes or pumps can be used to generate a pressure gradient that results in a force at the catheter tip, thereby establishing contact between the catheter and the clot (direct aspiration first-pass technique [ADAPT], Stent-retriever Associated Vacuum-locked Extraction [SAVE], and Aspiration-Retriever Technique for Stroke [ARTS] technique, and so forth) and generating backward flow to prevent clot embolization. ${ }^{3,6,11}$ The force at the catheter tip depends on only the catheter diameter and the applied pressure and is therefore not altered by resistors. ${ }^{12}$ The flow, however, is highly influenced by additional resistors; thus, any additional tubing and valves should be avoided whenever a backward flow is desired. Minimizing the resistance in the aspiration system is crucial because the necessary flow rate of aspirated blood is rather high: When the aim is to reverse blood flow, antegrade flow in the respective artery should be surpassed. In an average patient and without a balloon-guide catheter that restricts antegrade flow, aspirated blood flow must surpass $240 \mathrm{~mL} / \mathrm{min}$ in the ICA and $120 \mathrm{~mL}$ in the middle cerebral artery. ${ }^{13,14}$ Given that the flow through a 6F catheter even under ideal conditions (perfect vacuum, no external resistors, no stent retriever in its lumen) barely reaches $240 \mathrm{~mL} / \mathrm{min}$, we discourage using small guiding catheters for flow control in the ICA. ${ }^{5}$

In summary, the ideal aspiration system generates a high and constant pressure gradient and has minimal resistance. The latter is particularly important because our results show that pumps achieve similar pressure gradients but significantly differing effective flows because of their differing tubing systems. The resistance is not an inherent constant, but a coefficient that depends on variables such as flow speed and flow direction as well as fluid density and viscosity. However, because pump distributors do not provide any information about their tubing system, it is impossible for clinicians to estimate effective aspiration flow. We have quantified flow characteristics of various means of flow control and found significant differences among the methods.

\section{Sixty-Milliliter VacLok Vacuum Pressure Syringe}

The 60-mL syringe achieved high flows that surpassed blood flow in the ICA with an $8 \mathrm{~F}$ sheath and an $8 \mathrm{~F}$ balloon-guide catheter. With the newest generation of distal access catheters (ACE 64), aspiration flow was high enough to surpass blood flow in the MCA. This would even be the case with a stent retriever in the catheter, which reduces flow by approximately $15 \%{ }^{5}$ Syringes provide constant and high flow during an acceptable time (Fig 2), a finding that we did not expect because filling the syringe results in a loss of the pressure gradient. The nonlinear pressure loss, the resistors (syringe tip, 3-way valve, and hemostatic valve), and the inertia of the aspirated blood may be the main reasons for syringes achieving a comparably constant flow despite the unavoidable pressure loss during filling. Whether a syringe should be drawn gradually during the thrombectomy maneuver or drawn and locked before the maneuver has been a subject of discussion. Interventionalists, who draw syringes gradually during the thrombectomy maneuver, report that a tremble during manual aspiration (probably due to the Bernoulli effect) precedes collapse of the vessel and therefore helps anticipate it. However, insufficient flow remains an issue. In our experience, the average interventionalist easily generates approximately $50 \mathrm{kPa}(56 \%)$ of the maximal 90 $\mathrm{kPa}$ vacuum pressure by drawing the first $7 \mathrm{~mL}$ of a $60-\mathrm{mL}$ syringe. However, drawing a further $13 \mathrm{~mL}$ to achieve $75 \mathrm{kPa}$ and drawing the whole $60-\mathrm{mL}$ syringe for a maximal vacuum pressure of $90 \mathrm{kPa}$ are disproportionally more difficult. Hence, manual feedback when drawing the syringe may give a disproportionate impression of effective flow. Also, maintaining a specific pressure gradient without a pressure gauge is challenging. Furthermore, switching from one syringe to another results in a temporary flow arrest during mechanical thrombectomy. 


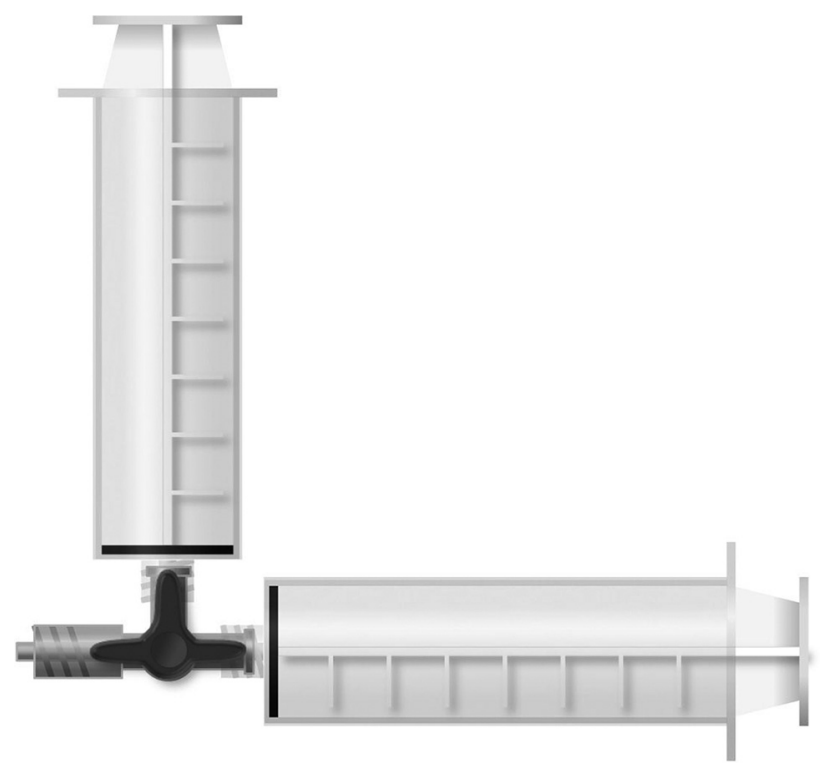

FIG 3. Syringe setup. To avoid flow arrest when switching from one syringe to another, one should connect 2 syringes in parallel to double aspiration volume and thus aspiration time. The 3-way valve, which is in a locked position, is then connected to the hemostatic $\mathrm{Y}$-adapter of the respective aspiration catheter. Before the thrombectomy maneuver starts, the syringes are fully drawn and locked to generate vacuum pressure, while the 3-way valve is still in a locked position. When the thrombectomy maneuver starts, the 3-way valve is set to be open for both syringes at the same time, thereby doubling aspiration volume and thus aspiration time.

Thus, for the sake of simplicity and safety of flow control, we advocate drawing and locking the syringes for aspiration. To avoid flow arrest when switching from one syringe to another, 2 syringes should be connected in parallel and drawn and locked before the thrombectomy maneuver to comfortably double aspiration volume and thus aspiration time (Fig 3). Attention should be paid to drawing the syringes immediately before the thrombectomy maneuver because we found, for our setup, a pressure loss of approximately $10 \mathrm{kPa}$ every 91 seconds or $6.6 \mathrm{kPa}$ per minute on average because the syringes and the valves are not perfectly sealed.

\section{Penumbra Pump}

To our surprise, the Penumbra pump achieved a rather low flow through an $8 \mathrm{~F}$ long sheath or an $8 \mathrm{~F}$ balloon-guide catheter and is therefore very unlikely to reverse blood flow in the ICA (On-line Table and Fig 1). This low flow is due to the high resistance of the tubing, which has a narrowing at the connection site and at the on/off switch, which are considerably smaller than the diameter of an $8 \mathrm{~F}$ catheter. The pump is dedicated for use with distal aspiration catheters; nonetheless, many interventionalists use it for aspiration in the ICA, a practice that is not only discouraged by the manufacturer but is also futile according to our experiments. Also, even with the newest generation of distal aspiration catheters (ACE 64), the Penumbra pump barely achieves flow that can reverse blood flow in the MCA. Thus, the pump with its current tubing should not be used for flow control but rather for techniques in which the force at the catheter tip, not the flow, is essential (for example, ADAPT). ${ }^{12}$

\section{Medela Pump}

The Medela pump achieved high flow that surpassed blood flow in the ICA with an $8 \mathrm{~F}$ sheath and an $8 \mathrm{~F}$ balloon-guide catheter. With the newest generation of distal access catheters (ACE 64), aspiration flow was high enough to surpass blood flow in the MCA, even with a stent retriever in the catheter, which reduces flow by approximately $15 \% .{ }^{5}$ Because high flow may cause vessel collapse, attention should be paid during aspiration.

\section{Limitations}

The major limitation of our study is the lack of experiments with blood and the limited number of investigated catheters. However, we deliberately chose to conduct a concise study, which captures the essential issues while keeping our results as simple and valid as possible, especially because data in the literature suggest that the viscosity of blood is altered contingently by the necessary anticoagulants. ${ }^{10} \mathrm{We}$ did not aspirate the water-glycerol solution in a model with tubes that match the diameters of the actual target vessels. Because the vessel diameter has an impact on possible flow volume, we chose to conduct our experiments with a reservoir to reduce the number of possible confounding factors. Hence, our results represent flow rates under ideal conditions; actual flow rates, in particular flow rates in small vessels such the M1 segment of the MCA of a patient, are likely to be even smaller.

\section{CONCLUSIONS}

Aspiration with syringes or pumps can be used to generate a pressure gradient with the following results: 1) a force at the catheter tip, thereby establishing contact between the catheter and the clot (ADAPT, SAVE, and ARTS technique, and so forth), or 2) backward flow to prevent clot embolization. The force at the catheter tip depends only on the catheter diameter and the applied pressure and is therefore not altered by resistors. The flow, however, is highly influenced by additional resistors. The ideal aspiration system generates a high and constant pressure gradient and has minimal resistance. The $60-\mathrm{mL}$ VacLok vacuum pressure syringe and the Medela pump achieved constant and high flows that are likely to be sufficient to reverse blood flow during thrombectomy with an $8 \mathrm{~F}$ sheath or balloon-guide catheter in the ICA and modern distal aspiration catheters in the MCA. The Penumbra pump is dedicated for use with distal aspiration catheters and should not be used with its current tubing for flow control in the ICA and MCA without proximal balloon protection.

\section{ACKNOWLEDGMENTS}

The authors thank Arash Nikoubashman for revising the manuscript.

Disclosures: Carolin Brockmann—UNRELATED: Employment: University Hospital Mainz, Germany. Martin Wiesmann—UNRELATED: Consultancy: Stryker; Payment for Lectures Including Service on Speakers Bureaus: Bracco, Medtronic, Siemens, Stryker; Payment for Development of Educational Presentations: Abbott, ab medica, Acandis, Bayer, Bracco, Braun, Codman, Medtronic, Dahlhausen, MicroVention, Penumbra, phenox, Philips Healthcare, Siemens, Silk Road, St. Jude, Stryker*; Support for Travel to Meetings for the Study or Other Purposes: Stryker, Comment: The study was funded by internal funds and we did not receive any support by Stryker. *Money paid to the institution. 


\section{REFERENCES}

1. Goyal M, Menon BK, van Zwam WH, et al; HERMES collaborators. Endovascular thrombectomy after large-vessel ischaemic stroke: a meta-analysis of individual patient data from five randomised trials. Lancet 2016;387:1723-31 CrossRef Medline

2. Chueh JY, Puri AS, Wakhloo AK, et al. Risk of distal embolization with stent retriever thrombectomy and ADAPT. J Neurointerv Surg 2016;8:197-202 CrossRef Medline

3. Maus V, Behme D, Kabbasch C, et al. Maximizing first-pass complete reperfusion with SAVE. Clin Neuroradiol 2017 Feb 13. [Epub ahead of print] CrossRef Medline

4. Wiesmann M, Brockmann MA, Heringer S, et al. Active push deployment technique improves stent/vessel-wall interaction in endovascular treatment of acute stroke with stent retrievers. J Neurointerv Surg 2017;9:253-56 CrossRef Medline

5. Nikoubashman O, Alt JP, Nikoubashman A, et al. Optimizing endovascular stroke treatment: removing the microcatheter before clot retrieval with stent-retrievers increases aspiration flow. J Neurointerv Surg 2017;9:459-62 CrossRef Medline

6. Massari F, Henninger N, Lozano JD, et al. ARTS (Aspiration-Retriever Technique for Stroke): initial clinical experience. Interv Neuroradiol 2016;22:325-32 CrossRef Medline

7. Chueh JY, Kuhn AL, Puri AS, et al. Reduction in distal emboli with proximal flow control during mechanical thrombectomy: a quantitative in vitro study. Stroke 2013;44:1396-401 CrossRef Medline

8. Jouvent E, Poupon C, Gray F, et al. Intracortical infarcts in small vessel disease: a combined 7-T postmortem MRI and neuropathological case study in cerebral autosomal-dominant arteriopathy with subcortical infarcts and leukoencephalopathy. Stroke 2011;42: e27-30 CrossRef Medline

9. Shih AY, Blinder P, Tsai PS, et al. The smallest stroke: occlusion of one penetrating vessel leads to infarction and a cognitive deficit. Nat Neurosci 2013;16:55-63 CrossRef Medline

10. Mayer GA, Kiss $\mathrm{O}$. Blood viscosity and in vitro anticoagulants. $A m \mathrm{~J}$ Physiol 1965;208:795-97 Medline

11. Hu YC, Stiefel MF. Force and aspiration analysis of the ADAPT technique in acute ischemic stroke treatment. J Neurointerv Surg 2016;8:244-46 CrossRef Medline

12. Nikoubashman O, Nikoubashman A, Büsen M, et al. Necessary catheter diameters for mechanical thrombectomy with ADAPT. AJNR Am J Neuroradiol 2017;38:2277-81 CrossRef Medline

13. Stock KW, Wetzel SG, Lyrer PA, et al. Quantification of blood flow in the middle cerebral artery with phase-contrast MR imaging. Eur Radiol 2000;10:1795-800 CrossRef Medline

14. Schöning M, Walter J, Scheel P. Estimation of cerebral blood flow through color duplex sonography of the carotid and vertebral arteries in healthy adults. Stroke 1994;25:17-22 CrossRef Medline 